\title{
Desempenho Reprodutivo de Novilhas de Corte Acasaladas aos 18 ou aos 24 Meses de Idade $^{1}$
}

\author{
Maurício Dallmann da Silva ${ }^{2}$, Júlio Otávio Jardim Barcellos ${ }^{3}$, Ênio Rosa Prates ${ }^{4}$
}

\begin{abstract}
RESUMO - O experimento foi desenvolvido com o objetivo de se avaliar duas idades de acasalamento, 18 meses (18M) e 24 meses (24M) em novilhas de corte Hereford, por monta natural, durante 45 dias. As variáveis analisadas foram a taxa de prenhez (TP), a condição corporal no início do acasalamento (CCI), o ganho de peso médio diário durante o período de acasalamento (GDM), a produtividade (P), representada pela quantidade de $\mathrm{kg}$ de bezerro desmamado por vaca acasalada, e a eficiência (E), ou seja, a produtividade para cada 100 $\mathrm{kg}$ de vaca. O período de acasalamento foi de 05/04/2000 a 20/05/2000 e de 04/11/2000 a 19/12/2000, para os grupos $18 \mathrm{M}$ e 24M, respectivamente. Novilhas do grupo 24M demonstraram maiores TP e CCI (86,7\% e 3,86) que as do grupo 18M (52,2\% e 3,59). O GDM nos períodos de acasalamento não apresentou diferença, sendo 0,728 e $0,724 \mathrm{~kg} / \mathrm{d}$, para $18 \mathrm{M}$ e $24 \mathrm{M}$, respectivamente. A P e a E das novilhas do grupo 24M foram superiores (135,2 e 38,6 kg) às do grupo 18M (76,2 e 26,6 kg, respectivamente). A redução da idade de acasalamento para os 18 meses requer maiores pesos no início do acasalamento para assegurar maiores índices reprodutivos.
\end{abstract}

Palavras-chave: acasalamento de outono, eficiência, produtividade, taxa de prenhez

\section{Reproductive Performance of Beef Heifers Bred at 18 or 24 Months of Age}

ABSTRACT - A 45-d experiment was conducted to evaluate two breeding ages of Hereford heifers, 18 (18M) or 24 months (24M), using natural mating. The following parameters were studied: pregnancy rate (PR), body condition score at the beginning of the mating season (BCS), and average daily gain in the mating season (ADG). It was also calculated the productivity $(\mathrm{P})=$ total $\mathrm{kg}$ of weaned calf per number of heifers bred and efficiency $(\mathrm{E})=$ productivity per $100 \mathrm{~kg}$ of heifers mated. The breeding seasons were from $04 / 05 / 2000$ to $05 / 20 / 2000$ (fall) and from $11 / 04 / 2000$ to $12 / 19 / 2000$ (spring) for $18 \mathrm{M}$ and $24 \mathrm{M}$ heifers, respectively. The $24 \mathrm{M}$ heifers showed higher PR and BCS than those bred at $18 \mathrm{M}$ of age $(86.7 \%$ vs. $52.2 \%$ and 3.86 vs. 3.59 , respectively). Conversely, breeding season did not affect the ADG comparing $24 \mathrm{M}(0.728 \mathrm{~kg} / \mathrm{d})$ with $18 \mathrm{M}(0.724 \mathrm{~kg} / \mathrm{d})$ heifers. The $24 \mathrm{M}$ heifers had greater P $(135.2 \mathrm{vs} .76 .2 \mathrm{~kg})$ and better E $(38.6 \mathrm{vs} .26 .6 \mathrm{~kg})$ than heifers mated at $18 \mathrm{M}$. It can be concluded that decreasing the breeding age from $24 \mathrm{M}$ to $18 \mathrm{M}$ requires higher body weight at beginning of the mating season in order to improve the reproductive parameters evaluated in the current trial.

Key Words: autumn breeding, pregnancy rate, productivity, eficiency

\section{Introdução}

Um sistema de produção de bovinos de corte que visa a sustentabilidade, baseada na viabilidade biológica e econômica, requer a compreensão de inúmeros fatores relacionados à produção, à implantação e utilização de novas tecnologias e práticas de manejo que estimulem o aumento da produtividade.

A idade ao primeiro acasalamento em bovinos de corte é uma variável que afeta a produtividade dos sistemas de cria, uma vez que reduz o número de novilhas em recria e (Potter et al., 1998) e aumenta a produtividade da vaca (Chapman et al., 1978). Contudo, esse aumento da produtividade deve ser acompanhado pelo retorno econômico resultante destas melhorias físicas do sistema (Faria, 1996; Barcellos et al., 2003).
Short et al. (1994) apresentam as principais vantagens e desvantagens de acasalar novilhas em idades mais precoces. Como vantagens, os autores relacionaram o retorno mais rápido do investimento, o aumento da vida produtiva de cada vaca e a menor relação entre reposição e reprodução. As desvantagens seriam a elevação dos custos para a novilha entrar em reprodução em idade mais jovem, o aumento da distocia, maiores investimentos em manejo para lidar com problemas de parto que as vacas com dois anos teriam e a menor taxa de retorno ao cio quando comparadas com vacas mais velhas.

Até o início da primeira temporada de acasalamento, as novilhas encontram-se em uma das etapas mais onerosas do ciclo de produção, pois esta categoria ainda nada produz e tem grandes exigências

\footnotetext{
1 Parte da Dissertação de Mestrado do primeiro autor apresentada à UFRGS - PPG/Zootecnia.

2 Médico Veterinário, MSc.

3 Professor, Doutor, Departamento de Zootecnia - UFRGS. E.mail: julio.barcellos@ufrgs.br

4 Professor Associado, PhD, Departamento de Zootecnia - UFRGS.
} 
nutricionais (Ferrel \& Jenkins, 1988; Rocha \& Lobato, 2002). Quando se busca a máxima eficiência biológica, deve-se fazer o acasalamento quando as novilhas estão com 14-15 meses de idade, porém, são necessários elevados aportes alimentares durante a criação da novilha, os quais normalmente têm custo elevado e muitas vezes não garantem desempenhos satisfatórios (Freitas \& Lobato, 2003).

O elevado custo desse tipo de alimentação envolve o uso de sistemas intensivos de criação, com utilização de pastagens cultivadas e suplementação alimentar estratégica. No estado do Rio Grande do Sul, em sistemas que utilizam somente pastagens nativas, dificilmente a novilha atinge o desenvolvimento necessário para o acasalamento aos 14 meses, pois, mesmo com cargas animais ajustadas e trabalhandose com vedação de piquetes, alguns trabalhos demonstraram baixos índices reprodutivos, que limitaram o desejado aumento da produtividade do sistema (Rocha \& Lobato, 2002; Freitas \& Lobato, 2003). Por outro lado, Eller et al. (2002) e Silva et al. (2003) em regiões tropicais, demonstraram a relevância do acasalamento da novilha aos 14 meses para a obtenção de maiores produtividades em sistemas de cria.

Como alternativa para reduzir a idade ao primeiro acasalamento dos 24 para os $14-15$ meses de idade, pode-se utilizar sistemas intermediários com o primeiro serviço aos 18 meses de idade, em uma outra estação reprodutiva (outono). Nesse sistema, os custos com a criação das novilhas são menores que os obtidos no sistema 14-15 meses. Além da diminuição dos custos para recria da desmama até o acasalamento, por meio da antecipação da temporada normal, obtém-se a redução das exigências nutricionais no segundo acasalamento (primavera), buscando-se melhor desempenho reprodutivo das primíparas, por não mais estarem lactando (Sampedro et al., 1995). Contudo, o aumento da utilização desta idade ao primeiro serviço, como prática de manejo, determinará a existência de dois rebanhos distintos em um mesmo sistema de cria, o de primavera e o de outono, o que pode ser considerado desvantagem ao sistema de produção. Além disso, os bezerros nascidos do acasalamento de outono apresentam menor peso à desmama que os de primavera (Barcellos et al., 1996).

Visando estudar a viabilidade dessa alternativa, este experimento foi desenvolvido com o objetivo de avaliar o desempenho reprodutivo, a produtividade e a eficiência de novilhas acasaladas aos 18 ou aos 24 meses de idade.

\section{Material e Métodos}

O experimento foi conduzido no município de Bagé, região fisiográfica da Campanha, no sudoeste do Rio Grande do Sul, situada entre os paralelos $30^{\circ}$ $30^{\prime}$ e $31^{\circ} 56^{\prime}$ latitude sul e os meridianos $55^{\circ} 30^{\prime}$ e $54^{\circ}$ $30^{\prime}$ longitude oeste de Greenwich. Segundo a classificação de Köppen, o clima da região é mesotérmico, tipo subtropical, da classe $\mathrm{Cfa}$, com chuvas regularmente distribuídas durante o ano. A precipitação média anual é de $1.350 \mathrm{~mm}$ com amplitude de $20 \%$. A distribuição das chuvas durante o ano situa-se em torno de $34 \%$ no inverno, $25 \%$ na primavera, $25 \%$ no outono e $16 \%$ no verão. A temperatura média anual é de $17,6^{\circ} \mathrm{C}$, sendo a média do mês mais quente (janeiro) de $24^{\circ} \mathrm{C}$ e a do mês mais frio (junho) de $12,5^{\circ} \mathrm{C}$. As temperaturas extremas são de $-4^{\circ} \mathrm{C}$ no mês mais frio e $41^{\circ} \mathrm{C}$ no mês mais quente (Moreno, 1961). A umidade relativa do ar oscila entre 75 e $85 \%$ e os ventos predominantes são sudeste, de setembro a abril e nordeste, de maio a agosto. Geadas ocorrem entre os meses de maio e setembro.

As pastagens são baixas e densas, com composição botânica diversificada e variações estacionais na qualidade e disponibilidade de forragem. A cobertura vegetal nos solos do local do experimento é superior a $80 \%$, sendo formada, principalmente, por campos considerados de boa qualidade e composta por grande número de espécies de gramíneas rizomatosas e/ou estoloníferas e boa freqüência de leguminosas. Essas espécies crescem especialmente na primavera/verão. Entre as gramíneas predominam a grama-forquilha (Paspalum notatum), o capim-melador (Paspalum dilatatum) e a grama-tapete (Axonopus compressus). As leguminosas encontradas são o pega-pega (Desmodium pratensis) e o trevo nativo (Tripholium polimorfum). As espécies hibernais são compostas principalmente por capim-flexilha (Stipa spp.).

Foram utilizadas 89 novilhas Hereford nascidas na primavera de 1998, originadas do rebanho comercial da propriedade, cuja idade ao acasalamento ocorre aos 24 meses de idade. Nesse rebanho, toda matriz apta à reprodução que está vazia no diagnóstico de gestação é sumariamente eliminada do programa de cria. Essas novilhas foram separadas aleatoriamente em dois grupos experimentais em 05 de abril de 2000. Um grupo foi constituído de 46 novilhas para o acasalamento aos 18 meses de idade (18M), que ocorreu no outono (05/04 - 20/05/2000), e o outro, de 
43 novilhas para o acasalamento aos 24 meses de idade (24M), na primavera/verão (04/11 - 19/12/ 2000). O acasalamento, em ambos os grupos, ocorreu por monta natural, utilizando-se dois touros em cada grupo, todos com os mesmos valores esperados na progênie para a característica de peso à desmama. Dentro dos grupos, a diferença de idade das novilhas era de, no máximo, 60 dias, podendo variar, no grupo $18 \mathrm{M}$, de 17 até 19 meses de idade e, no 24M, de 23 até 25 meses.

As novilhas foram pesadas no início (PIA) e no final de cada temporada de acasalamento, permitindo medir o ganho de peso diário médio durante o acasalamento (GDM). A cada pesagem, as novilhas foram submetidas a uma avaliação visual de sua condição corporal (CC), conforme os critérios adaptados da classificação de Lowman et al. (1973), em que $\mathrm{CC}=1$ muito magro e $\mathrm{CC}=5$ muito gordo.

Durante as temporadas de acasalamento, foram coletadas amostras da pastagem, pelo método descrito por t'Mannetje (1978), para medir a disponibilidade e a qualidade da forragem disponível.

Os bezerros nascidos no final do verão de 2000 e da primavera de 2001, filhos das novilhas dos grupos $18 \mathrm{M}$ e do grupo $24 \mathrm{M}$, respectivamente, foram desmamados em 08 de setembro e 15 de março, com idades médias de 210 e 200 dias, respectivamente.

$O$ cálculo dos índices de produtividade $(\mathrm{P})$ para as duas idades de acasalamento foi baseado no procedimento descrito por Baker \& Carter (1976), em que: Produtividade $=($ número de bezerros desmamados $*$ peso médio dos bezerros à desmama) / número de novilhas acasaladas. Neste experimento, para o cálculo da produtividade, foram considerados o número de novilhas acasaladas, o número de bezerros desmamados e o peso médio à desmama ajustado para a idade padrão de 205 dias.

A eficiência (E) dos dois sistemas de acasalamento também foi calculada segundo Baker \& Carter (1976), em que: Eficiência $=$ Produtividade $/($ Peso da vaca/100).

Como os dados e os erros experimentais de $\mathrm{P}$ e E não apresentaram uma distribuição normal, pela ocorrência do valor zero atribuído às novilhas que não conceberam, estes sofreram uma transformação logarítimica $y=\ln (x+0,5)$, sendo $y$ a variável transformada e $x$ a variável na escala original (SPSS, 2002).

Uma análise preliminar, dentro de cada grupo, não demonstrou efeitos da idade real da novilha sobre os parâmetros estudados.
A porcentagem de prenhez (TP) foi calculada com base no diagnóstico de gestação por palpação retal, feito 90 dias após o término do período de monta, sendo comparada entre os dois grupos experimentais (18M e 24M) pelo teste Qui-quadrado (SPSS, 2002).

A comparação entre tratamentos quanto às variáveis contínuas foi feita pelo teste $t$, utilizando-se o programa estatístico Statgraphics 4.1 (1999).

\section{Resultados e Discussão}

Na Tabela 1 constam os resultados de peso no início do acasalamento (PIA) e da taxa de prenhez (TP) dos grupos experimentais.

Observou-se diferença significativa da TP entre ambos os grupos (idades) $(\mathrm{P}<0,05), 18 \mathrm{M}$ e $24 \mathrm{M}$, com superioridade para novilhas acasaladas aos 24 meses (Tabela 1), o que sugere que a idade exerceu influência sobre o desempenho reprodutivo. Contudo, Chapman et al., (1978), comparando as taxas de prenhez de novilhas Hereford de 15 ou 24 meses, acasaladas com pesos semelhantes, não observaram efeitos da idade sobre os parâmetros estudados, o que evidencia claramente ser o peso vivo a variável mais importante na manifestação da atividade reprodutiva em novilhas (Hess et al., 2005). Morrison et al. (1992), ao conduzirem um experimento semelhante ao trabalho em análise, com novilhas nascidas no outono ou na primavera para atingir o primeiro parto com 24 ou 30 meses de idade, observaram, ao final do período reprodutivo, semelhante porcentagem de prenhez para ambasas idades. É provável que as diferenças na TP tenham decorrido principalmente das diferenças de

\begin{tabular}{|c|c|c|c|}
\hline \multirow{2}{*}{$\begin{array}{l}\text { Idade (meses) } \\
\text { Age (months) }\end{array}$} & Grupo & PIA (kg) & ТP (\%) \\
\hline & Group & $B W(k g)$ & $P R(\%)$ \\
\hline \multirow{2}{*}{$\begin{array}{l}18 \\
24\end{array}$} & $18 \mathrm{M}$ & $286,7^{\mathrm{b}}$ & $52,2^{\mathrm{B}}$ \\
\hline & $24 \mathrm{M}$ & $350,6^{\mathrm{a}}$ & $86,7^{\mathrm{A}}$ \\
\hline
\end{tabular}

a,b: Médias seguidas de letras diferentes na coluna diferem $(P<0,05)$ entre si pelo teste $t$.

$a, b$ : Means followed by different letters, in the same column, differ $(P<0.05)$ by $t$ - test.

$A, B$ : Médias seguidas de letras diferentes na coluna diferem $(\mathrm{P}<0,05)$ entre si pelo teste $\chi^{2}$.

$A, B$ : Means followed by different letters, in the same column, differ $(P<0.05)$ by $\chi^{2}$ test. 
peso no início do acasalamento de cada grupo de novilhas, embora uma análise em cada grupo não tenha demonstrado diferenças no PIA entre as novilhas que conceberam e as que falharam ( $289 \mathrm{vs} 283 \mathrm{~kg}$ e 349 vs $355 \mathrm{~kg}$, para $18 \mathrm{M}$ e $24 \mathrm{M}$, respectivamente).

A maioria dos pesquisadores, ao comparar efeitos de idade de acasalamento, restringe-se em avaliar novilhas aos 14-15 meses em relação aos 24 meses, que é a idade usual do primeiro acasalamento de novilhas de corte no Brasil. Os resultados deste trabalho, com novilhas acasaladas aos 18 meses demonstraram menor taxa de prenhez nesta idade, provavelmente em razão dos efeitos da estação do ano ou do peso vivo.

A maior taxa de prenhez $(\mathrm{P}<0,05)$ do grupo $24 \mathrm{M}$ confirma os resultados obtidos por Pereira Neto \& Lobato (1998), comprovando que há um efeito marcante do peso ao início do acasalamento sobre a taxa de prenhez. Peso no início do acasalamento equivalente a $65 \%$ do peso da vaca adulta, em raças britânicas, pode assegurar elevados índices de prenhez no primeiro acasalamento (Bolze \& Corah, 1993).

Sampedro et al. (1995), trabalhando com novilhas 2/3 Hereford - 1/3 Brahman acasaladas aos 18 meses de idade, no outono, obtiveram taxas de prenhez de $80 \%$, muito superiores aos $52,2 \%$ observados neste trabalho. Entretanto, os animais experimentais utilizados por esses autores eram de menor frame e provavelmente com exigência de menores pesos no início do acasalamento.

Neste estudo, os PIA dos grupos $18 \mathrm{M}$ e $24 \mathrm{M}$ representavam, respectivamente, 59 e $73 \%$ dos pesos médios das vacas adultas, que, no local do experimento, eram de $480 \mathrm{~kg}$. O valor percentual foi maior no grupo acasalado aos 24 meses de idade e superior aos valores descritos por Bolze \& Corah (1993) como pesos críticos para o primeiro acasalamento. No entanto, o grupo $18 \mathrm{M}$ apresentou percentual do peso da vaca adulta menor que o sugerido na literatura (NRC, 1996), havendo possibilidade de prejuízo no desempenho reprodutivo. Silva (2003), ao comparar o desempenho reprodutivo de dois grupos de novilhas Hereford acasaladas aos 18 meses, no outono, com pesos médios de 301 e $264 \mathrm{~kg}$, observou taxa de prenhezde 73,3 e $26,7 \%$, respectivamente, evidenciando que, no grupo $18 \mathrm{M}(286 \mathrm{~kg})$, ocorreu uma limitação no desenvolvimento das novilhas para essa idade de acasalamento. Portanto, o fato de a maioria das novilhas do grupo $18 \mathrm{M}$ não ter alcançado o peso mínimo, associado à possível imaturidade fisiológica de algumas delas, pode ter limitado o desempenho reprodutivo e dificultado uma análise mais aprofundada das possíveis diferenças entre as duas idades de acasalamento.

Neste trabalho, as temporadas de acasalamento utilizadas foram de apenas 45 dias de duração, compreendendo, neste período, somente dois ciclos estrais. É provável que, em uma estação reprodutiva mais prolongada, com o aumento do peso vivo e com maior maturidade das novilhas, as possibilidades de aparecimento de estros seriam superiores (Cohen et al., 1980), resultando em maior taxa de prenhez. Como isso não ocorreu, a taxa de prenhez nas novilhas acasaladas aos 18 meses foi baixa e insuficiente para uma produtividade mais elevada em relação à das novilhas com 24 meses. A utilização de períodos curtos de acasalamento visa concentrar a parição e alcançar maior homogeneidade dos produtos. Contudo, pode limitar o desempenho reprodutivo de novilhas cujos pesos vivos encontram-se em um limiar inferior ao exigido no início do acasalamento, especialmente na estação outonal (Barcellos et al., 2000).

Os efeitos da época do ano não foram considerados no desenho experimental, pois o pressuposto básico da fêmea bovina é ser poliéstrica anual, não havendo influências das estações do ano sobre a fertilidade, desde que o sistema alimentar seja semelhante (Patterson et al., 1992; Schillo et al., 1992).

$\mathrm{Na}$ Tabela 2 estão apresentados os resultados de condição corporal no início do acasalamento (CCI) dos grupos experimentais.

As novilhas acasaladas aos 24 meses de idade (24M) apresentavam melhores CCI $(\mathrm{P}<0,05)$ que as do grupo $18 \mathrm{M}$. Quanto à CCI observada no grupo $18 \mathrm{M}$, não foram encontrados resultados em novilhas Hereford com mesma idade, porém, Semmelmann et al. (2001) obtiveram, em novilhas Nelore aos 18 meses de idade, CCI semelhantes às encontradas neste trabalho. Contudo, esse é um parâmetro de menor relevância na avaliação do desenvolvimento de novilhas jovens, pois, em animais em crescimento, as diferenças existentes na condição corporal são de difícil visualização e, por isso, muitas vezes, não são encontradas relações entre a CCI e o desempenho reprodutivo (Brooks et al., 1985; Beretta \& Lobato, 1998; Vargas et al., 1999).

É importante ressaltar que tanto o PIA quanto a CCI foram avaliações pontuais na ocasião do início 
Tabela 2 - Condição corporal no início do acasalamento $(\mathrm{CCl})$ e ganho diário médio (GDM) segundo o grupo experimental

Table 2 - Body condition score (BCS) and average daily gain (ADG) according to experimental group

\begin{tabular}{lccc}
\hline Idade (meses) & Grupo & CCI $(1-5)$ & GDM $(\mathrm{kg})$ \\
Age (months) & Group & $B C S(1-5)$ & $A D G(k g)$ \\
\hline 18 & $18 \mathrm{M}$ & $3,59^{\mathrm{b}}$ & $0,728^{\mathrm{a}}$ \\
24 & $24 \mathrm{M}$ & $3,86^{\mathrm{a}}$ & $0,724^{\mathrm{a}}$ \\
\hline
\end{tabular}

$a, b$ : Médias seguidas de letras diferentes na coluna diferem $(P<0,05)$ entre si pelo teste $t$.

$a, b$ : Means followed by different letters, in the same column, differ $(P<0.05)$ by $t$ - test.

das temporadas de acasalamento. A partir dessas datas, ocorreram modificações no estado nutricional dos animais, resultantes das variações de peso (GDM) observadas durante o período de 45 dias, por ocasião do acasalamento, as quais podem ter alterado o escore da condição corporal até o final do período reprodutivo.

Os resultados (Tabela 2) indicam que não houve diferença significativa $(\mathrm{P}>0,05)$ no GDM entre os 18 e 24 meses de idade. Também não ocorreram diferenças no GDM, dentro de cada grupo, entre as novilhas que conceberam e as que falharam, o que evidencia a ausência de relação entre essa variável e a taxa de prenhez. Esses GDM são considerados altos para o outono e a primavera, por ocasião das épocas de acasalamento, evidenciando o potencial das pastagens naturais da região onde foi desenvolvido o experimento.

Quanto ao GDM do grupo 18M, não foram identificados resultados de experimentos semelhantes que permitissem uma comparação com os ganhos obtidos neste trabalho. Portanto, a discussão apresentada refere-se a experimentos desenvolvidos a partir de outros protocolos.

Semmelmann et al. (2001), trabalhando com novilhas Nelore, acasalando-as aos 18 meses de idade no outono, em pastagens de braquiária (Brachiaria brizanta $\mathrm{Cv}$. Marandu), no estado de São Paulo, obteve GDM de 0,481 kg durante a temporada de acasalamento. No grupo acasalado na primavera, os GDM foram superiores aos obtidos em animais acasalados na mesma idade e época, manejados em campo nativo no Rio Grande do Sul (Almeida \& Lobato, 2004).

$\mathrm{Na}$ Tabela 3 são apresentados os resultados de produtividade e eficiência das duas idades de acasalamento.
Tabela 3 - Produtividade $(P)$ e eficiência $(E)$ de novilhas de corte, de acordo com a idade ao acasalamento

Table 3 - Productivity $(P)$ and efficiency $(E)$ of beef heifers according to breeding age

\begin{tabular}{lccc}
\hline $\begin{array}{l}\text { Idade (meses) } \\
\text { Age (months) }\end{array}$ & $\begin{array}{c}\text { Grupo } \\
\text { Group }\end{array}$ & $\mathrm{P}(\mathrm{kg})$ & $\mathrm{E}(\mathrm{kg})$ \\
\hline 18 & $18 \mathrm{M}$ & $76,2^{\mathrm{b}}$ & $26,6^{\mathrm{b}}$ \\
24 & $24 \mathrm{M}$ & $135,2^{\mathrm{a}}$ & $38,6^{\mathrm{a}}$ \\
\hline
\end{tabular}

a,b: Médias seguidas de letras diferentes na coluna diferem $(P<0,05)$ entre si pelo teste $t$.

$a, b$ : Means followed by different letters, in the same column, differ $(P<0.05)$ by $t$ - test.

A análise comprovou efeitos da idade ao primeiro acasalamento sobre os parâmetros de produtividade e eficiência.

O grupo 24M apresentou maior produtividade $(\mathrm{P}<0,05)$ que o grupo $18 \mathrm{M}$, em decorrência da maior taxa de prenhez e também do maior peso à desmama dos seus bezerros, produzindo, então, maior número de quilogramas de bezerros desmamados por novilha exposta. Quando avaliada a eficiência das novilhas, também foi encontrada diferença significativa $(\mathrm{P}<0,05)$ entre as idades; apesar de as novilhas do grupo 24M serem mais pesadas, foram mais eficientes para cada $100 \mathrm{~kg}$ de peso vivo que as $18 \mathrm{M}$.

Os resultados obtidos neste trabalho são semelhantes aos registrados por Barcellos et al., (1996), que, avaliando primíparas, obtiveram resultados médios para eficiência de aproximadamente $30 \mathrm{~kg}$ de bezerros desmamados para cada $100 \mathrm{~kg}$ de vaca exposta. Por outro lado, a produtividade e a eficiência foram superiores aos resultados encontrados por Ribeiro \& Lobato (1988), que foram, em média, de $46,9 \mathrm{~kg}$ e $11,7 \mathrm{~kg}$, respectivamente.

\section{Conclusões}

A antecipação da idade ao primeiro acasalamento de 24 para 18 meses de idade é viável biologicamente, porém, para obtenção de melhores índices reprodutivos no acasalamento aos 18 meses, são necessárias melhorias na fase de recria das fêmeas bovinas, para que alcancem maior peso vivo no primeiro acasalamento.

O primeiro acasalamento aos 18 meses, em razão das menores taxas de prenhez, apresenta menor produtividade e eficiência no sistema de cria que o acasalamento aos 24 meses. 


\section{Literatura Citada}

BAKER, R.L.; CARTER, A.H. Influence of breed and crossbreeding on beef cow performance. In: RUAKURA FARMERS CONFERENCE, 1976, Hamilton. Proceedings... Hamilton: 1976. v.9, p.39-44.

BARCELLOS, J.O.J.; LOBATO, J.F.P.; FRIES, L.A. Eficiência de vacas primíparas Hereford e cruzas Hereford-Nelore acasaladas no outono/inverno ou na primavera/verão. Revista Brasileira de Zootecnia, v.25, n.3, p.414-427, 1996.

BARCELlOS, J.O.J.; PATIÑO, H.O.; PRATES, E.R. et al. Carga animal pós-desmama e desempenho reprodutivo de novilhas de corte acasaladas aos 18 meses de idade. In: REUNIÃO ANUAL DA SOCIEDADE BRASILEIRA DE ZOOTECNIA, 37., 2000, Viçosa, MG. Anais... Viçosa: Sociedade Brasileira de Zootecnia, 2000. CD-ROM.

BARCELlOS, J.O.J.; COSTA, E.C.; SILVA, M.D. et al. Crescimento de fêmeas bovinas de corte aplicado aos sistemas de cria. Sistemas de produção em bovinos de corte. Porto Alegre: Universidade Federal do Rio Grande do Sul, 2003. 72p. (Publicação Ocasional, 1)

BERETTA, V.; LOBATO, J.F.P. Sistema “um ano" de produção de carne: avaliação de estratégias alternativas de alimentação hibernal de novilhas de reposição. Revista Brasileira de Zootecnia, v.27, n.1, p.157-163, 1998.

BOLZE, R.; CORAH, L.R. Selection and development of replacement heifers. Manhattan: Kansas State University. Cooperative Extension Service, 1993. 9p.

BROOKS, A.L.; MORROW, R.E.; YOUNGQUIST, R.S. Body composition of beef heifers at puberty. Theriogenology, v.24, n.2, p.235-250, 1985.

COHEN, R.D.H.; GARDNER, D.L.; LANGLANDS, J.P. A note on the relationship between live weight and incidence of oestrous in Hereford heifers. Animal Production, v.31, p.221-222, 1980.

CHAPMAN, H.D.; YOUNG, J.M.; MORRISON, E.G. et al. Differences in lifetime productivity of Hereford calving first at 2 and 3 years of age. Journal of Animal Science, v.46, n.5, p.1159-1162, 1978.

ELER, J.P.; SILVA, J.A.V.; FERRAZ, J.B.S. et al. Genetic evaluation of the probability of pregnancy at 14 months for Nellore heifers. Journal of Animal Science, v.80, n.4, p.951-954, 2002.

FARIA, N.R. Grandes projetos agropecuários - carne - sistemas de produção, custos, comercialização. Revista Brasileira de Reprodução Animal, v.20, n.2, p.106-111, 1996.

FERREL, C.L.; JENKINS, T.G. Influence of biological types on energy requirements. Agricultural Research Service: United States Department of Agriculture, 1988. p.86-90. (Beef Research Progress Report 3)

FREITAS, S.G.; LOBATO, J.F.P. Desempenho reprodutivo e produtivo de novilhas de corte aos dois anos de idade submetidas a diferentes alternativas de alimentação. In: REUNIÃO ANUAL DA SOCIEDADE BRASILEIRA DE ZOOTECNIA, 40., 2003, Santa Maria. Anais... Santa Maria: Sociedade Brasileira de Zootecnia, 2003. CD-ROM.

HESS, B.W.; LAKE, S.L.; SCHOLLJEGERDES, E.J. et al. Nutritional controls of beef cow reproduction. Journal
Animal Science, v.83, (E.Suppl.), p.E90-E106, 2005

LOWMAN, B.G.; SCOTT, N.; SOMERVILLE, S. Condition scoring beef cattle. Edinburgh: East of Scotland College of Agriculture, 1973. 8p. (Bulletin, 6)

ALMEIDA, L.S.P.; LOBATO, J.F.P. Efeito da idade de desmame e suplementação no desemvolvimento de novilhas de corte. Revista Brasileira de Zootecnia, v.33, n.6, p.2086-2094, 2004 (Supl. 2).

MORENO, J.A. Clima do Rio Grande do Sul. Porto Alegre: Secretaria da Agricultura, 1961. 42p.

MORRISON, D.G.; FEAZEL, J.I.; BAGLEY, C.P. et al. Postweaning growth and reproduction of beef heifers exposed to calve at 24 or 30 months of age in spring and fall seasons. Journal of Animal Science, v.70, n.3, p.622-630, 1992.

NATIONAL RESEARCH COUNCIL - NRC. Nutrients requirements of cattle. Washington, D.C.: National Academy Press, 1996. 242p.

PATTERSON, D.J.; PERRY, R.C.; KIRACOFE, G.H. et al. Management consideration in heifer development and puberty. Journal Animal Science, v.70, n.12, p.4018-4035, 1992.

PEREIRA NETO, O.A.; LOBATO, J.F.P. Efeitos da ordem de utilização de pastagens nativas melhoradas no desenvolvimento e comportamento reprodutivo de novilhas de corte. Revista Brasileira de Zootecnia, v.27, n.1, p.60-65, 1998.

POTTER, L.; LOBATO, J.F.P.; NETTO, C.G.M. Produtividade de um modelo de produção para novilhas de corte primíparas aos dois, três ou quatro anos de idade. Revista Brasileira de Zootecnia, v.27, n.3, p.613-619, 1998.

RIBEIRO, A.M.L.; LOBATO, J.F.P. Produtividade e eficiência reprodutiva de três grupos raciais de novilhas de corte. I. Desempenho reprodutivo. Revista Brasileira de Zootecnia, v.17, p.498-507, 1988.

ROCHA, M.G.; LOBATO, J.F.P. Avaliação do desempenho reprodutivo de novilhas de corte primíparas aos dois anos de idade. Revista Brasileira Zootecnia, v.31, n.3, p.13881395, Supl., 2002.

SAMPEDRO, D.; VOGEL, O.; CELSER, R. Alternativas de manejo para entorar la vaquilla a los 18 meses de edad: $\mathrm{Su}$ influencia sobre el porcentaje de $2^{\mathrm{O}}$ entore y prenhez. Mercedes: INTA, 1995. 9p. (Circular Técnica).

SCHILLO, K.K.; HALL, J.B.; HILEMAN, S.M. Effects of nutrition and season on the onset of puberty in beef heifer. Journal Animal Science, v.70, n.12, p.39944005, 1992.

SEMMELMANN, C.E.N.; LOBATO, J.F.P.; ROCHA, M.G. Efeito de sistemas de alimentação no ganho de peso e desempenho reprodutivo de novilhas Nelore acasaladas aos 17-18 meses. Revista Brasileira de Zootecnia, v.30, n.3, p.835-843, 2001.

SHORT, R.E.; STAIGMILLER, R.B.; BELLOWS, R.A. et al. Breeding heifers at one year of age: biological and economic considerations. In: FIELDS, M.J.; SAND, R.S. (Eds.) Factors Affecting calf crop. Boca Raton: CRC Press, 1994. p.55-68.

SILVA, M.D. Desempenho reprodutivo de novilhas de corte acasaladas aos 18 ou 24 meses de idade. Porto Alegre: Universidade Federal do Rio Grande do Sul, 2003. 107p. Dissertação (Mestrado em Zootecnia) - Universidade Federal do Rio Grande do Sul, 2003.

SILVA, J.A. II V.; MELIS, M.H.; ELER, J.P. et al. Estimação de 
parâmetros para probabilidade de prenhez aos 14 meses e altura na garupa em bovinos da raça Nelore. Revista Brasileira de Zootecnia, v.32, n.5, p.1141-1146, 2003.

SPPS User's guide: Statistics. SPSS Inc Version 11.5. Headquarters. Chicago. IL. 2002.

STATGRAPHICS: User's Manual Version 4.1 for Windows. Statistical Graphics Corporation, 1999.

t'MANNETJE, L. Measuring quantity of grassland vegetation. In: t'MANNETJE, L. (Ed.) Measurement of grassland vegetation and animal production. Farnham Royal: Commonwealth Agricultural Bureaux, 1978. p.63-90. (Bulletin, 52)

Recebido em: 23/10/03

Aceito em: 30/06/05

VARGAS, C.A.; OLSON, T.A.; CHASE, C.C. et al. Influence of frame size and body condition score on performance of Brahman cattle. Journal of Animal Science, v.77, n.12, (dic), p.3140-3149, 1999. 